\title{
The Study on Urban Landscape Suitability Index of Indigenous Arbors, Shrubs In Nanchang
}

\author{
Xu Qin ${ }^{1}$ \\ ${ }^{1}$ Landscape Architecture Department, Nanchang Institute of Technology, Nanchang, Jiangxi, China
}

\begin{abstract}
This paper through brainstorming, the Delphi method, and in-meeting \& after-meeting method, screening out suitability evaluation indicators of indigenous arbors, shrubs plants for urban landscapes in Nanchang. It sets up the suitability evaluation indicator system of indigenous arbors, shrubs to urban landscapes in Nanchang. To improve bio-diversity in cities, we need to focus on indigenous plants in greening initiatives. In this study, the indigenous plants in Nanchang were investigated to analyze the current situations of indigenous plants in this region and their application in urban greening in Nanchang. The problems in using indigenous plants for greening in Nanchang City were analyzed and corresponding suggestions were made.
\end{abstract}

\section{Introduction}

The species composition of arbors and shrubs can reflect the vegetation types of a region, and the plant community of urban landscapes, which must be dominated by native plants, should be formed by simulating the natural community structure according to the plant flora of the city. The plant configuration should be dominated by native plants while supplemented by exotic plants, so as to ensure species diversity and landscape diversity, which is the basis for maintaining biodiversity. There are abundant native resources of arbors and shrubs in Nanchang, but there are still few species used in urban greening. How many kinds of them are suitable for urban ecological environment and available for urban greening? For this question, it is necessary to put forward a method for screening excellent native arbors and shrubs so as to provide sufficient basis for the rational selection and application of native plants in urban landscaping.

\section{Resources and Methods}

\subsection{Data Collection and Sorting}

The species of indigenous arbors and shrubs in Nanchang studied in this paper are all derived from the author's field survey. The route survey and the special survey are combined to investigate the peripheral and internal areas of Nanchang urban jurisdiction. The urban periphery includes Meiling Forest Park, Shengshuitang Forest Park and Xiangshan Forest Park, and the urban interior mainly refers to Yaohu Forest Botanical Park, Aixi Lake Wetland Park, Bayi Park, People's Park and other large parks, as well as residential areas, enterprises and institutions and major urban roads with relatively better greening. The investigation contents include plant species, characteristics, habitats and ornamental characteristics, etc., which are investigated through field investigation and inductive statistics, as well as synchronous photography, recording and filing. According to Flora of Jiangxi ${ }^{[1]}$ [2] [3] $^{[3}$, it is concluded after investigation that there are 129 families, 317 genera and 451 species of native plants in Nanchang, including 191 species of indigenous arbors and shrubs.

\subsection{Research Methods}

\subsubsection{Establishment of the Suitability Indexes of Indigenous Arbors and Shrubs for Urban Landscapes}

The brainstorming method, the Delphi method, and the in-meeting and after-meeting method are combined to screen out the suitability evaluation indexes of indigenous arbors and shrubs for urban landscapes and to establish the suitability evaluation index system of indigenous arbors and shrubs for urban landscapes.

\subsubsection{A Study of the Suitability Evaluation System of Indigenous Arbors and Shrubs for Urban Landscapes in Nanchang}

With indigenous arbors and shrubs in Nanchang as the objects, the suitability evaluation indexes of indigenous arbors and shrubs for urban landscapes are stratified by expert scoring method, the weight of each evaluation index on the suitability of indigenous arbors and shrubs for urban landscapes in Nanchang is determined by the analytic hierarchy process, and the suitability evaluation index system of indigenous arbors and shrubs for urban 
landscapes in Nanchang is established. By calculating the score value of each index and the product of its weight of the suitability evaluation system of indigenous arbors and shrubs for urban landscapes in Nanchang and by obtaining the accumulated value, the suitability indexes of indigenous arbors and shrubs for urban landscapes in Nanchang are obtained. According to the suitability indexes of indigenous arbors and shrubs for urban landscapes in Nanchang, the indigenous arbors and shrubs in Nanchang are divided into the four grades: I, II, III and IV, indicating the excellent, the good, the common and the poor level, respectively ${ }^{[4]}$.

\section{Setting Up the Evaluation System}

\subsection{The Suitability Evaluation Index System of Indigenous Arbors and Shrubs for Urban Landscapes in Nanchang}

Through collecting and sorting out the domestic research literature related to indigenous arbors and shrubs, combined with the research contents of forest cities and landscape aesthetics, a total of 30 primary, secondary and tertiary evaluation indexes are collected. Ten relevant experts are invited to combine the brainstorming method, the Delphi method and the in-meeting and after-meeting method to screen out the evaluation indexes of the suitability of indigenous arbors and shrubs for urban landscapes in Nanchang. The target layer is the suitability of indigenous arbors and shrubs for urban landscapes in Nanchang. There are four indexes in the criterion layer and 20 indexes in the scheme layer. The evaluation index system of the suitability of indigenous arbors and shrubs for urban landscapes in Nanchang is determined. See Table 1.

Tab. 1 The Evaluation Index System of the Suitability of Indigenous Arbors and Shrubs for Urban Landscapes in Nanchang

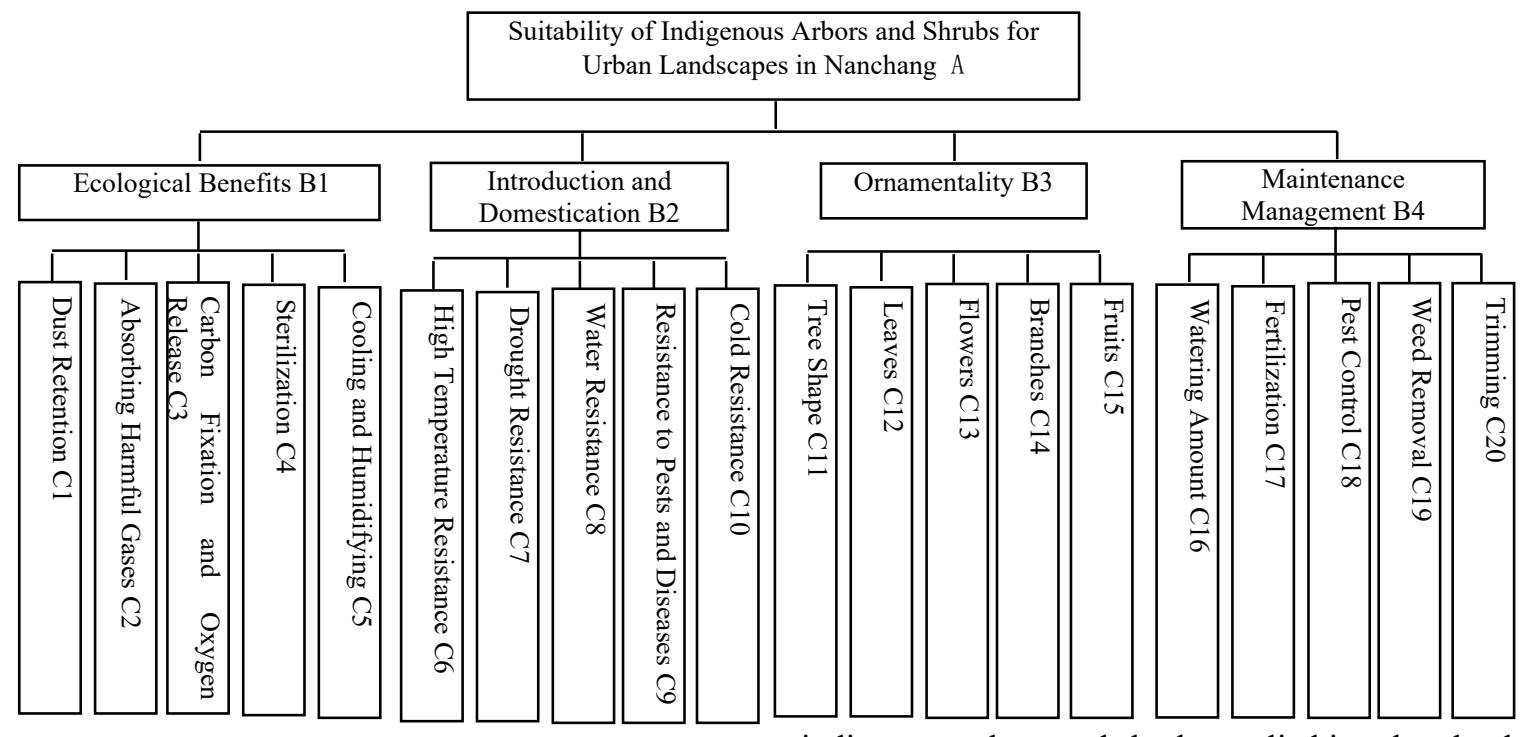

\subsection{Calculating the Suitability Indexes of Indigenous Arbors and Shrubs for Urban Landscapes in Nanchang}

The suitability of indigenous arbors and shrubs for urban landscapes is measured according to the suitability evaluation indexes of indigenous arbors and shrubs for urban landscapes. By calculating the score values of the 24 indexes of the suitability evaluation system of indigenous arbors and shrubs for urban landscapes and the products of their weights, their accumulated values are defined as the suitability indexes of indigenous arbors and shrubs for urban landscapes. The suitability of indigenous arbors and shrubs applied in urban landscapes is measured according to the suitability index of indigenous arbors and shrubs for urban landscapes.

The suitability indexes of indigenous arbors and shrubs for urban landscapes are set as the percentage system, and are attached to different scores according to the weight of each index. On this basis, according to the suitability indexes of indigenous arbors and shrubs for urban landscapes, the suitability of indigenous arbors and shrubs for urban landscapes is divided into five grades: I, II, III and IV, indicating the excellent, the good, the common and the poor level, respectively. The division results are shown in Table 2. 
Tab. 2 Division standard table of indigenous plants suitability index rank in urban landscape

\begin{tabular}{ccc}
\hline $\begin{array}{c}\text { Grades of Indigenous Arbors and } \\
\text { Shrubs }\end{array}$ & $\begin{array}{c}\text { Grades of the Suitability Indexes of Indigenous Arbors and Shrubs } \\
\text { for Urban Landscapes }\end{array}$ & Remarks \\
\hline I & $90-100$ & Excellent \\
II & $70-90$ & Good \\
Common \\
III & $60-70$ & Poor \\
\hline
\end{tabular}

\subsection{A Study of the Suitability Evaluation Index System of Indigenous Arbors and Shrubs for Urban Landscapes in Nanchang}

\subsubsection{Constructing the Judgment Matrix}

Through practicing the expert investigation on the experts from Jiangxi Provincial Forestry Department,

Tab. 3 Judgement matrix (B1-C)

\begin{tabular}{lcccc}
\hline & Ecological Benefits & $\begin{array}{c}\text { Introduction and } \\
\text { Domestication }\end{array}$ & Ornamentality & $\begin{array}{c}\text { Maintenance } \\
\text { Management }\end{array}$ \\
\hline Ecological Benefits & 1 & 1 & 2 & 3 \\
Introduction and Domestication & 1 & 1 & 2 & 2 \\
Ornamentality & 0.5 & 0.5 & 1 & 2 \\
Maintenance Management & 0.333333333 & 0.5 & 0.5 & 1 \\
\hline
\end{tabular}

Tab. 4 Judgement matrix (B1-C)

\begin{tabular}{|c|c|c|c|c|c|}
\hline & Dust Retention & $\begin{array}{c}\text { Absorbing Harmful } \\
\text { Gases }\end{array}$ & $\begin{array}{c}\text { Fixation and } \\
\text { Oxygen Release }\end{array}$ & Sterilization & $\begin{array}{l}\text { Cooling and } \\
\text { Humidifying }\end{array}$ \\
\hline Dust Retention & 1 & 2 & 2 & 3 & 2 \\
\hline Absorbing Harmful Gases & 0.5 & 1 & 2 & 2 & 2 \\
\hline $\begin{array}{l}\text { Carbon Fixation and } \\
\text { Oxygen Release }\end{array}$ & 0.5 & 0.5 & 1 & 2 & 2 \\
\hline Sterilization & 0.333333333 & 0.5 & 0.5 & 1 & 0.5 \\
\hline Cooling and Humidifying & 0.5 & 0.5 & 0.5 & 2 & 1 \\
\hline
\end{tabular}

Tab. 5 Judgement matrix (B2-C)

\begin{tabular}{|c|c|c|c|c|c|}
\hline & $\begin{array}{c}\text { High Temperature } \\
\text { Resistance }\end{array}$ & Drought Resistance & $\begin{array}{c}\text { Water } \\
\text { Resistance }\end{array}$ & $\begin{array}{l}\text { Resistance to Pests } \\
\text { and Diseases }\end{array}$ & Cold Resistance \\
\hline Water Resistance & 1 & 0.5 & 1 & 2 & 1 \\
\hline $\begin{array}{l}\text { Resistance to Pests } \\
\text { and Diseases }\end{array}$ & 0.5 & 1 & 0.5 & 1 & 0.5 \\
\hline
\end{tabular}

Tab. 6 Judgement matrix (B3-C)

\begin{tabular}{lccccc}
\hline & Tree Shape & Leaves & Flowers & Branches & Fruits \\
\hline Tree Shape & 1 & 2 & 2 & 3 & 3 \\
Leaves & 0.5 & 1 & 1 & 2 & 2 \\
Flowers & 0.5 & 1 & 1 & 2 & 1 \\
Branches & 0.333333333 & 0.5 & 0.5 & 1 & 2 \\
Fruits & 0.333333333 & 0.5 & 1 & 0.5 & 1 \\
\hline
\end{tabular}

Tab. 7 Judgement matrix (B4-C)

\begin{tabular}{|c|c|c|c|c|c|}
\hline & Watering Amount & Fertilization & Pest Control & $\begin{array}{c}\text { Weed } \\
\text { Removal }\end{array}$ & Trimming \\
\hline Watering Amount & 1 & 2 & 2 & 2 & 2 \\
\hline Pest Control & 0.5 & 0.5 & 1 & 2 & 1 \\
\hline Weed Removal & 0.333333333 & 0.5 & 0.5 & 1 & 2 \\
\hline
\end{tabular}




\subsubsection{Hierarchical Ranking and Consistency Checking}

The feature vectors of the criterion layer corresponding to the target layer and the scheme layer corresponding to the criterion layer are obtained by each judgment matrix, and then the weight is calculated by the feature root method, and the consistency of the results of the total weight is checked. The results are shown in Table 8.

Tab. 8 Consistency Checking of Hierarchical Single Sequence

\begin{tabular}{llllll}
\hline Single Sequencing & A-Bn & B1-Cn & B2-Cn & B3-Cn & B4-Cn \\
\hline$\lambda$ max & 4.045692 & 5.145981 & 5.38494 & 5.153899 & 5.157475 \\
$\mathrm{CR}$ & 0.016923 & 0.032585 & 0.085924 & 0.034352 & 0.035151 \\
\hline
\end{tabular}

\subsubsection{Total Hierarchy Permutation}

quantified. The percentage system (upper limit exclusion

According to the above relationship of the hierarchical structure, the comprehensive weight of the suitability of indigenous arbors and shrubs for urban landscapes in method) is used to determine the score value of each index, each index is divided into three grades, and each grade is given a certain score. The results are shown in Table 9. Nanchang is calculated, and each index is weighted and

Tab. 9 Total Hierarchy Permutation of the Suitability of Indigenous Arbors and Shrubs for Urban Landscapes in Nanchang

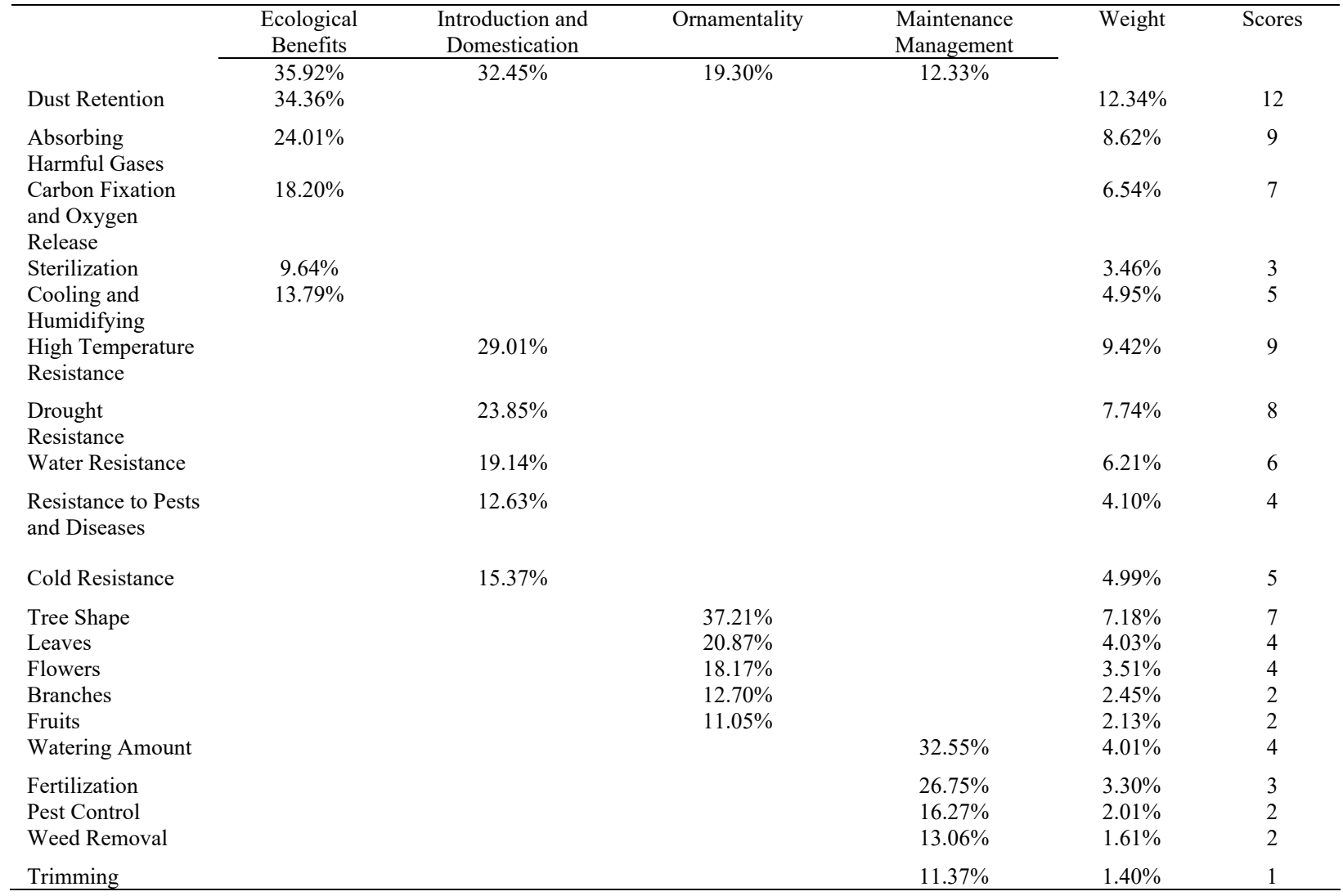

\section{Results and Discussion}

After a total of 191 indigenous arbors and shrubs in Nanchang are assigned values and calculated, there are a total of 104 species with a comprehensive score above 60 points, as detailed in table 10. Among them, except those that have been used in gardens, the evergreen arbors include Schima superba, Altingia chinensis, Cyclobalanopsis glauca, Cyclobalanopsis jenseniana, Lithocarpus glaber, Tutcheria hirta, Elaeocarpus duclouxii, Castanopsis fargesii, Castanopsis sclerophylla, etc., the deciduous arbors include Vernicia fordii, Betula luminifera, Carpinus viminea, Castanea seguinii and Quercus variabilis, etc., the evergreen shrubs include Rosa laevigata, Rosa cymosa, Sycopsis sinensis, Turpinia arguta, Ilex chinensis, Symplocos sumuntia, ndocalamus latifolius, etc., and the deciduous shrubs include Lespedeza davidii, Lespedeza formosa, Dalbergia mimosoides, Lespedeza cuneata, Rhododendron ovatum, etc.. A large number of native species with potentials in application are waiting for development and application.

Nanchang is located in the evergreen broad-leaved forest area of the subtropical monsoon climate, so the urban greening should be dominated by evergreen species. In order to protect biodiversity, the backbone tree species and the keynote tree species should be mainly native tree species, so as to improve plant diversity and create regional characteristic landscape. However, at present, the utilization rate of indigenous arbors and shrubs in the backbone tree species and keynote tree species in Nanchang is relatively low, and 
foreign tree species are dominant in various green spaces such as roads, squares, parks and residential areas and so on, which needs to be improved urgently.

Tab. 10 The Mark Sheet of the Suitability Indexes of Indigenous Arbors and Shrubs for Urban Landscapes in Nanchang

\begin{tabular}{|c|c|c|c|}
\hline Specific Name & Latin Name & $\begin{array}{l}\text { Comprehensiv } \\
\text { e Evaluation }\end{array}$ & $\begin{array}{l}\text { Grad } \\
\text { e }\end{array}$ \\
\hline Masson pine & Pinus massoniana & 91 & $\mathrm{I}$ \\
\hline Chinese fir & $\begin{array}{l}\text { Cunninghamia } \\
\text { lanceolata }\end{array}$ & 92 & I \\
\hline Camphor tree & Sassafras tzumu & 90 & I \\
\hline $\begin{array}{l}\text { Common } \\
\text { sassafras }\end{array}$ & Sassafras tzumu & 91 & I \\
\hline $\begin{array}{l}\text { Leatherleaf } \\
\text { mahonis }\end{array}$ & Mahonia bealei & 91.5 & I \\
\hline $\begin{array}{l}\text { Heavenly } \\
\text { bamboo }\end{array}$ & $\begin{array}{l}\text { Nandina } \\
\text { domestica }\end{array}$ & 92.5 & I \\
\hline Tea oil camellia & Camellia oleifera & 92 & I \\
\hline Tea & Camellia sinensis & 95 & I \\
\hline Schima & Schima superba & 95 & I \\
\hline $\begin{array}{l}\text { Chinese St. } \\
\text { John's wort }\end{array}$ & $\begin{array}{l}\text { Hypericum } \\
\text { monogynum }\end{array}$ & 92.5 & I \\
\hline $\begin{array}{l}\text { Elaeocarpus } \\
\text { glabripetalus } \\
\text { Merr. }\end{array}$ & $\begin{array}{l}\text { Elaeocarpus } \\
\text { glabripetalus }\end{array}$ & 90 & $\mathrm{I}$ \\
\hline $\begin{array}{l}\text { Chinese tallow } \\
\text { tree }\end{array}$ & Sapium sebiferum & 91.5 & I \\
\hline Tungoiltree & Vernicia fordii & 92 & I \\
\hline David bush & Lespedeza davidii & 91.5 & I \\
\hline Altlngia & Altingia chinensis & 91 & I \\
\hline $\begin{array}{l}\text { Beautiful } \\
\text { sweetgum }\end{array}$ & $\begin{array}{l}\text { Liquidambar } \\
\text { formosana }\end{array}$ & 94 & I \\
\hline Dry willow & Salix matsudana & 93 & I \\
\hline $\begin{array}{l}\text { Chinese } \\
\text { waxmyrtle }\end{array}$ & Myrica rubra & 91 & I \\
\hline $\begin{array}{l}\text { Seguin } \\
\text { chinkapin cup }\end{array}$ & Castanea seguinii & 91.5 & I \\
\hline $\begin{array}{l}\text { Boue Japanese } \\
\text { oak }\end{array}$ & $\begin{array}{l}\text { Cyclobalanopsis } \\
\text { glauca }\end{array}$ & 92 & I \\
\hline $\begin{array}{l}\text { Cyclobalanopsis } \\
\text { jensenniana }\end{array}$ & $\begin{array}{l}\text { Cyclobalanopsis } \\
\text { jenseniana }\end{array}$ & 92 & I \\
\hline Pasania & $\begin{array}{l}\text { Lithocarpus } \\
\text { glaber }\end{array}$ & 91 & I \\
\hline Cork oak & $\begin{array}{l}\text { Quercus } \\
\text { variabilis }\end{array}$ & 92 & I \\
\hline Hackberry & Celtis sinensis & 94 & I \\
\hline Chinese elm & Ulmus parvifolia & 92 & I \\
\hline Paper mulberry & $\begin{array}{l}\text { Broussonetia } \\
\text { papyrifera }\end{array}$ & 90.5 & I \\
\hline Holly & Ilex chinensis & 90 & I \\
\hline Tea-of -heaven & $\begin{array}{l}\text { Ailanthus } \\
\text { altissima }\end{array}$ & 90 & I \\
\hline Chinaberry & Melia azedarach & 91 & I \\
\hline Chinese sumac & Rhus chinensis & 92.5 & I \\
\hline Chinese wingnut & $\begin{array}{l}\text { Pterocarya } \\
\text { stenoptera }\end{array}$ & 96 & I \\
\hline Azalea & $\begin{array}{l}\text { Rhododendron } \\
\text { simsii }\end{array}$ & 90 & I \\
\hline $\begin{array}{l}\text { Sumuntia } \\
\text { sweetleaf root }\end{array}$ & $\begin{array}{l}\text { Symplocos } \\
\text { sumuntia }\end{array}$ & 90.5 & I \\
\hline Chinese privet & Ligustrum sinense & 95.5 & I \\
\hline Cape jasmine & $\begin{array}{l}\text { Gardenia } \\
\text { jasminoides }\end{array}$ & 94.5 & I \\
\hline June snow herb & Serissa japonica & 95.5 & I \\
\hline Williams elder & $\begin{array}{l}\text { Sambucus } \\
\text { williamsii }\end{array}$ & 90.5 & I \\
\hline $\begin{array}{l}\text { Hempleaf } \\
\text { negundo } \\
\text { chastetree }\end{array}$ & $\begin{array}{l}\text { Vitex negundo var. } \\
\text { cannabifolia }\end{array}$ & 90.5 & I \\
\hline
\end{tabular}

\begin{tabular}{|c|c|c|c|}
\hline Specific Name & Latin Name & $\begin{array}{l}\text { Comprehensiv } \\
\text { e Evaluation }\end{array}$ & $\begin{array}{l}\text { Grad } \\
\text { e }\end{array}$ \\
\hline $\begin{array}{l}\text { Red-fruit } \\
\text { greyblue } \\
\text { spicebush }\end{array}$ & $\begin{array}{l}\text { Lindera } \\
\text { erythrocarpa }\end{array}$ & 71 & II \\
\hline $\begin{array}{l}\text { Mountain spicy } \\
\text { tree }\end{array}$ & Litsea cubeba & 75.5 & II \\
\hline $\begin{array}{l}\text { White-flower } \\
\text { longflower } \\
\text { stringbush }\end{array}$ & $\begin{array}{l}\text { Wikstroemia } \\
\text { trichotoma }\end{array}$ & 74 & II \\
\hline $\begin{array}{l}\text { Rough } \\
\text { brushwood }\end{array}$ & Tutcheria hirta & 88 & II \\
\hline $\begin{array}{l}\text { Elaeocarpus } \\
\text { duclouxii } \\
\text { gagnep }\end{array}$ & $\begin{array}{l}\text { Elaeocarpus } \\
\text { duclouxii }\end{array}$ & 87 & II \\
\hline Salem-rose herb & Rubus rosifolius & 70.5 & II \\
\hline $\begin{array}{l}\text { Mimoselike } \\
\text { rosewood }\end{array}$ & $\begin{array}{l}\text { Dalbergia } \\
\text { mimosoides }\end{array}$ & 81.5 & II \\
\hline $\begin{array}{l}\text { Cuneate } \\
\text { bush-clover } \\
\text { rootor herb }\end{array}$ & $\begin{array}{l}\text { Lespedeza } \\
\text { cuneata }\end{array}$ & 83.5 & II \\
\hline $\begin{array}{l}\text { Beautiful } \\
\text { lespedeza }\end{array}$ & $\begin{array}{l}\text { Lespedeza } \\
\text { formosa }\end{array}$ & 86.5 & II \\
\hline $\begin{array}{l}\text { Stachyurus } \\
\text { chinensis Franch }\end{array}$ & $\begin{array}{l}\text { Stachyurus } \\
\text { chinensis }\end{array}$ & 79.5 & II \\
\hline $\begin{array}{l}\text { Euonymus } \\
\text { japonicus }\end{array}$ & $\begin{array}{l}\text { Buxus } \\
\text { megistophylla }\end{array}$ & 78.5 & II \\
\hline $\begin{array}{l}\text { Betula } \\
\text { luminifera }\end{array}$ & Betula luminifera & 84.5 & II \\
\hline $\begin{array}{l}\text { Carpinus } \\
\text { viminea Wall }\end{array}$ & Carpinus viminea & 88.5 & II \\
\hline Oat chestnut & $\begin{array}{l}\text { Castanopsis } \\
\text { fargesii }\end{array}$ & 88.5 & II \\
\hline $\begin{array}{l}\text { Bitter } \\
\text { evergreenchinkapi } \\
\mathrm{n}\end{array}$ & $\begin{array}{l}\text { Castanopsis } \\
\text { sclerophylla }\end{array}$ & 80. & II \\
\hline Quercus alba & Quercus fabri & 70 & II \\
\hline Mulberry tree & Morus alba & 80 & II \\
\hline $\begin{array}{l}\text { Purple tephrosia } \\
\text { Oriental } \\
\text { bittersweet }\end{array}$ & $\begin{array}{l}\text { Celastrus } \\
\text { glaucophyllus }\end{array}$ & 72.5 & II \\
\hline $\begin{array}{l}\text { Oriental } \\
\text { buckthorn }\end{array}$ & Rhamnus crenata & 74.5 & II \\
\hline $\begin{array}{l}\text { Picrasma } \\
\text { quassioides } \quad \text { (D. } \\
\text { Don) Benn. }\end{array}$ & $\begin{array}{l}\text { Picrasma } \\
\text { quassioides }\end{array}$ & 80 & II \\
\hline Oliver maple & Acer oliverianum & 74 & II \\
\hline Acerwilsonii & Acer wilsonii & 79 & II \\
\hline $\begin{array}{l}\text { Euscaphis } \\
\text { japonica }\end{array}$ & $\begin{array}{l}\text { Euscaphis } \\
\text { japonica }\end{array}$ & 72.5 & II \\
\hline Wax-tree & $\begin{array}{l}\text { Toxicodendron } \\
\text { succedaneum }\end{array}$ & 75 & II \\
\hline Lacquer tree & $\begin{array}{l}\text { Toxicodendron } \\
\text { vernicifluum }\end{array}$ & 73 & II \\
\hline Dyetree & $\begin{array}{l}\text { Platycarya } \\
\text { strobilacea }\end{array}$ & 84 & II \\
\hline Spinystem aralia & $\begin{array}{l}\text { Aralia } \\
\text { echinocaulis }\end{array}$ & 71 & II \\
\hline Chinese aralia & Aralia elata & 74 & II \\
\hline $\begin{array}{l}\text { Dentiferous } \\
\text { dendropanax }\end{array}$ & $\begin{array}{l}\text { Dendropanax } \\
\text { dentigerus }\end{array}$ & 73 & II \\
\hline $\begin{array}{l}\text { Rhododendron } \\
\text { chrysodoron } \\
\text { ovatum }\end{array}$ & $\begin{array}{l}\text { Rhododendron } \\
\text { ovatum }\end{array}$ & 85 & II \\
\hline Purpus privet & Ligustrum quihoui & 88.5 & II \\
\hline Serissa herb & $\begin{array}{l}\text { Serissa } \\
\text { serissoides }\end{array}$ & 73 & II \\
\hline Moso bamboo & $\begin{array}{l}\text { Phyllostachys } \\
\text { edulis }\end{array}$ & 73 & II \\
\hline $\begin{array}{l}\text { Red poison } \\
\text { hemlock }\end{array}$ & $\begin{array}{l}\text { Illicium } \\
\text { lanceolatum }\end{array}$ & 68 & III \\
\hline $\begin{array}{l}\text { Greyblue } \\
\text { spicebush }\end{array}$ & Lindera glauca & 68 & III \\
\hline $\begin{array}{l}\text { Glabrous } \\
\text { scrcandra herb }\end{array}$ & Sarcandra glabra & 61.5 & III \\
\hline
\end{tabular}




\begin{tabular}{|c|c|c|c|}
\hline Specific Name & Latin Name & $\begin{array}{l}\text { Comprehensiv } \\
\text { e Evaluation }\end{array}$ & $\begin{array}{l}\text { Grad } \\
\mathrm{e}^{-}\end{array}$ \\
\hline $\begin{array}{l}\text { Boxleaf } \\
\text { syzygium }\end{array}$ & $\begin{array}{l}\text { Syzygium } \\
\text { buxifolium }\end{array}$ & 65.5 & III \\
\hline Twelvestamen & Melastoma & & III \\
\hline Melastoma herb & dodecandrum & 64.5 & \\
\hline Rose mallow & Urena lobata & 62.5 & III \\
\hline $\begin{array}{l}\text { Mountain } \\
\text { tallowtree }\end{array}$ & Sapium discolor & 62.5 & III \\
\hline $\begin{array}{l}\text { Deutzia } \\
\text { setchuenensis }\end{array}$ & $\begin{array}{l}\text { Deutzia } \\
\text { setchuenensis }\end{array}$ & 63.5 & III \\
\hline $\begin{array}{l}\text { Panicle } \\
\text { hydrangea }\end{array}$ & $\begin{array}{l}\text { Hydrangea } \\
\text { paniculata }\end{array}$ & 69.5 & III \\
\hline $\begin{array}{l}\text { Photinia } \\
\text { beauverdiana }\end{array}$ & $\begin{array}{l}\text { Photinia } \\
\text { beauverdiana }\end{array}$ & 62.5 & III \\
\hline $\begin{array}{l}\text { Everlasting } \\
\text { thorn }\end{array}$ & $\begin{array}{l}\text { Pyracantha } \\
\text { fortuneana }\end{array}$ & 69.5 & III \\
\hline Cheroke rose & Rosa laevigata & 64.5 & III \\
\hline Smallfruit rose & Rosa cymosa & 69.5 & III \\
\hline $\begin{array}{l}\text { Loropetalum } \\
\text { chinense }\end{array}$ & $\begin{array}{l}\text { Loropetalum } \\
\text { chinense }\end{array}$ & 69.5 & III \\
\hline Sycopsis & Sycopsis sinensis & 68.5 & III \\
\hline Raisin tree & $\begin{array}{l}\text { Hovenia } \\
\text { trichocarpa }\end{array}$ & 64 & III \\
\hline $\begin{array}{l}\text { Medicinal } \\
\text { evodia }\end{array}$ & $\begin{array}{l}\text { Evodia } \\
\text { rutaecarpaTetradi } \\
\text { um ruticarpum }\end{array}$ & 67.5 & III \\
\hline $\begin{array}{l}\text { Redwood } \\
\text { branches }\end{array}$ & $\begin{array}{l}\text { Meliosma } \\
\text { oldhamii }\end{array}$ & 63.5 & III \\
\hline $\begin{array}{l}\text { Common } \\
\text { craibiodendron }\end{array}$ & $\begin{array}{l}\text { Meliosma } \\
\text { rhoifolia } \\
\text { barbulata }\end{array}$ & 60.5 & III \\
\hline Turpinia arguta & Turpinia arguta & 61.5 & III \\
\hline & Alangium kurzii & 68 & III \\
\hline $\begin{array}{l}\text { Medicinal } \\
\text { evodia } \\
\text { slenderstyle }\end{array}$ & $\begin{array}{l}\text { Acanthopanax } \\
\text { evodiaefolius }\end{array}$ & & III \\
\hline acanthopanax & & 61 & \\
\hline Chinese ivy & $\begin{array}{l}\text { Hedera } \\
\text { nepalensis var. } \\
\text { sinensis }\end{array}$ & 64 & III \\
\hline $\begin{array}{l}\text { Whiteflower } \\
\text { tarenna }\end{array}$ & $\begin{array}{l}\text { Tarenna } \\
\text { mollissima }\end{array}$ & 67 & III \\
\hline Chinese abelia & Abelia chinensis & 64.5 & III \\
\hline $\begin{array}{l}\text { Fortune } \\
\text { paulownia }\end{array}$ & $\begin{array}{l}\text { Paulownia } \\
\text { fortunei }\end{array}$ & 64 & III \\
\hline $\begin{array}{l}\text { Purple } \\
\text { beautyberry }\end{array}$ & $\begin{array}{l}\text { Callicarpa } \\
\text { bodinieri }\end{array}$ & 68 & III \\
\hline $\begin{array}{l}\text { Girald } \\
\text { beautyberry }\end{array}$ & $\begin{array}{l}\text { Callicarpa } \\
\text { giraldii }\end{array}$ & 64 & III \\
\hline $\begin{array}{l}\text { Koch } \\
\text { beautyberry }\end{array}$ & $\begin{array}{l}\text { Callicarpa } \\
\text { kochiana }\end{array}$ & 68 & III \\
\hline $\begin{array}{l}\text { Manyflower } \\
\text { glorybower }\end{array}$ & $\begin{array}{l}\text { Clerodendrum } \\
\text { cyrtophyllum }\end{array}$ & 63 & III \\
\hline $\begin{array}{l}\text { Japanese premna } \\
\text { herb }\end{array}$ & $\begin{array}{l}\text { Premna } \\
\text { microphylla }\end{array}$ & 65 & III \\
\hline $\begin{array}{l}\text { Negundo } \\
\text { chastetree }\end{array}$ & Vitex negundo & 66.5 & III \\
\hline $\begin{array}{l}\text { Indocalamus } \\
\text { latifolius }\end{array}$ & $\begin{array}{l}\text { Indocalamus } \\
\text { latifolius }\end{array}$ & 66.5 & III \\
\hline
\end{tabular}

\section{Acknowledgments}

This paper is one of the phased achievements of science and technology research project 'Study on the Suitability Index of Indigenous Arbors for Urban Landscapes in Nanchang' (GJJJ161107) of Department of Education in Jiangxi Province.

\section{References}

1. Lin Y. (1993) Flora of Jiangxi (Volume 1). Jiangxi Science and Technology Press, Nanchang.

2. Editorial Board of Flora of Jiangxi. (2004) Flora of Jiangxi (Volume 2). China Science and Technology Press, Beijing.

3. Editorial Board of Flora of Jiangxi. (2014) Flora of Jiangxi (Volume 3). Jiangxi Science and Technology Press, Nanchang.

4. Xu Q. (2013) A Study of Suitability Index of Indigenous Plants for Urban Landscape in Changsha. Central South University of Forestry and Technology, Changsha. 